\title{
Partial trisomy 22 (q11.2-q13.1) as a result of duplication and pericentric inversion
}

\author{
V P Prasher, E Roberts, A Norman, A C Butler, V H R Krishnan, D J McMullan
}

\begin{abstract}
A case of a 27 year old male with a duplication of part of the long arm of chromosome 22 (22q11.2-q13.1) together with a pericentric inversion of the same chromosome is reported. Particular phenotypic features of note include absence of speech, persistent self-injury, lack of daily living skills, colobomata, and very poor vision. Similarities between this case and other case reports of duplications of the long arm of chromosome 22 are discussed.
\end{abstract}

( $($ Med Genet 1995;32:306-308)

Complete trisomy 22 has very rarely been reported in live births, ${ }^{12}$ although it is a common finding in spontaneous abortions. ${ }^{34}$ The existence of such a chromosomal abnormality has, however, been questioned ${ }^{5}$ although there have been a number of recent reports of trisomy 22 confirmed by in situ hybridisation techniques. ${ }^{67}$ Partial trisomies for both the proximal and distal segments of the long arm of chromosome 22 have been described by a number of authors. ${ }^{8-10}$ We report a case of a duplication of part of the long arm of chromosome 22 (22q11.2-q13.1) together with a

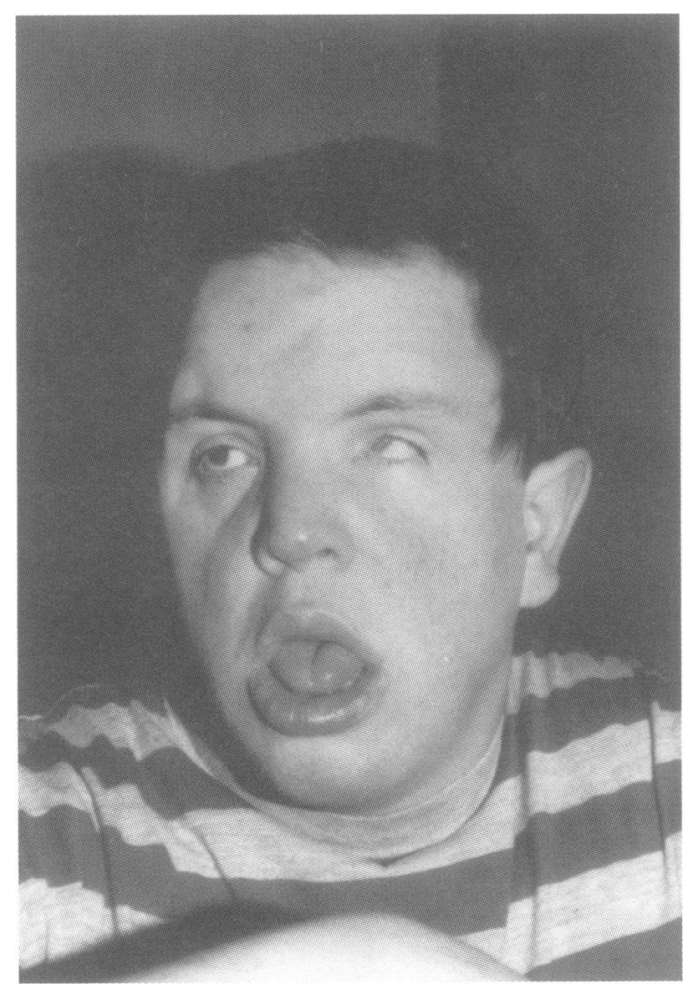

Figure 1 AP view of craniofacial dysmorphism of proband. pericentric inversion of the same chromosome.

\section{Case report}

The proband was a 27 year old male who had a moderate degree of learning disability and at the time of reporting was residing in a long stay unit for people with such impairment. Pregnancy and delivery had been normal, and although he was cared for at home for the first 11 years of his life, he spent subsequent years in long stay residential units. He had limited communication skills, poor mobility, and required help with all daily living skills. There was a longstanding history of persistent selfinjury and hyperoral activity.

On physical examination he was less than $150 \mathrm{~cm}$ tall $(<3 \mathrm{rd}$ centile) and weighed $70 \mathrm{~kg}$ $(<50$ th centile). His head circumference was $52 \mathrm{~cm}$, proportional for his height. He had a prominent forehead, low posterior hairline, prognathism of the lower jaw, tongue protrusion, and thick fleshy lips. In both eyes he had ectopic pupils with "pear shaped" colobomata with an abnormally pigmented retina and persistent pupillary membrane remnants. Vision was extremely poor in both eyes. His ears and nose appeared normal (figs 1 and 2).

$\mathrm{He}$ had bilateral single transverse palmar

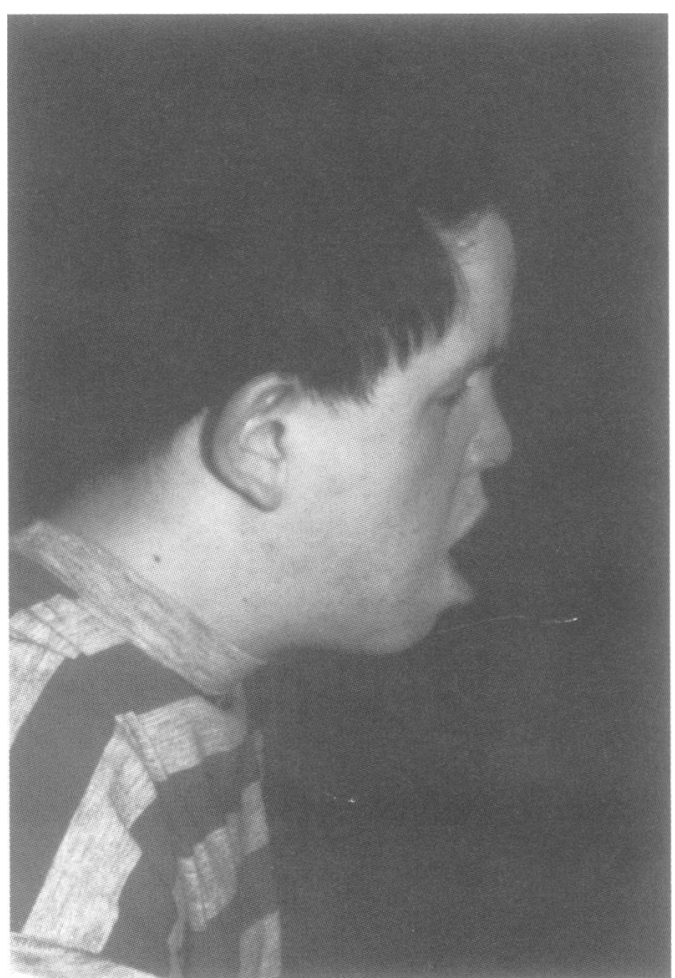

Figure 2 Lateral view of craniofacial dysmorphism of proband. 
A

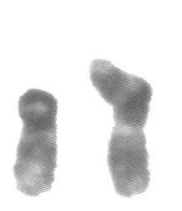

B
C

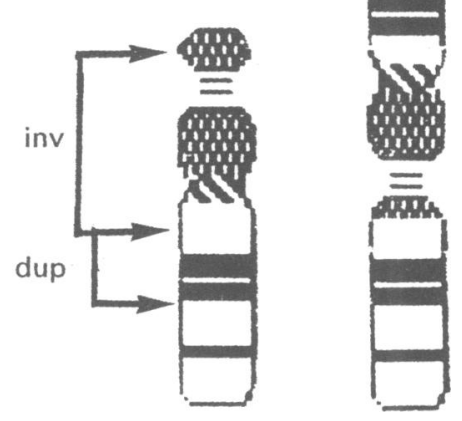

Figure 3 Partial karyotype of proband illustrating normal (left) and abnormal (right) chromosomes 22: (A,B) G banding, (C) schematic idiogram.

creases with short hands and fingers; the distal phalanx of both thumbs was particularly short. He also had short feet and toes. There was a moderate thoracic kyphosis. The distribution of body hair was normal with a normal male pattern of pubic hair growth, although he had a small penis and testes.

He had three sibs, aged 32,31 , and 19 years, who were well. There was no family history of any mental or physical disorder. $\mathrm{He}$ was prescribed no medication and had no psychiatric disorder associated with his learning disability.

\section{CYTOGENETICS}

Cytogenetic preparations were obtained from phytohaemagglutinin stimulated lymphocytes from a peripheral blood sample. Cultures were set up and harvested by standard methods.

$G$ banded analysis showed the presence of one normal chromosome 22 and a submetacentric chromosome of approximately the size of chromosome 16 . C banding confirmed the presence of only a single centromere, while staining with silver nitrate ${ }^{11}$ showed the presence of a nucleolus organiser region (NOR) in the middle of the long arm of the rearranged chromosome. Chromosome painting with a 14; 22 centromeric probe (D22Z3, Oncor) and with a chromosome 22 paint (Cambio) confirmed that all of the additional material was derived from chromosome 22 .

The rearranged chromosome was interpreted as resulting from a duplication (probably an inverted duplication) of part of the long arm of chromosome 22 (from q11.2 to q13.1) followed by a pericentric inversion, although other interpretations are also possible. The karyotype of the proband is therefore $46, \mathrm{XY}, \operatorname{dup}(22)$ (q11.2 $\rightarrow$ q13.1), inv(22) (p13q11.2) (figs 3 and $4)$. $\mathrm{He}$ is effectively trisomic for the region $22 \mathrm{q} 11.2$ to $22 \mathrm{q} 13$. Both parents have normal karyotypes.

A cell line has not been established from this patient.

Figure 4 Partial karyotype of proband, illustrating normal (left and abnormal (right) chromosome 22; (top) $C$ banding, (bottom) NOR staining.

\section{Discussion}

The majority of reported duplications of chromosome 22 involve the proximal segment of chromosome 22. Duplications of the distal segment have been reported in only a few cases, ${ }^{581213}$ but these cases have allowed the delineation of a distal $22 \mathrm{q}$ syndrome. Our patient appears to represent a duplication of $22 q 11.1$ to q13, which has only been reported once previously to our knowledge. ${ }^{14}$

The phenotypic features of the present case have a number of similarities with the case of Taylor et al, ${ }^{14}$ notably absence of speech, persistent self-injury, lack of daily living skills, and very poor vision. The case of Taylor et $a l^{14}$ has some features not present in our case, which may be accounted for by (1) slight differences in the apparent breakpoints or (2) a larger duplication in the former.

However, the phenotypic features in our patient also overlap to some extent those reported in a case by Abeliovich et $a l^{8}$ and the duplication present in that case may also overlap the region duplicated in the present case. As in that case, our patient represents a "pure" duplication of $22 \mathrm{q}$, in this case from $\mathrm{q} 11.2$ to q13.1, without involvement of any other chromosome segment. It is noteworthy that both our patient and that reported by $\mathrm{Ab}$ eliovich $e t a l^{8}$ have colobomata, which has been commonly reported in the "cat eye" syndrome (assigned to 22pter $\rightarrow \mathrm{q} 11$ ). ${ }^{15}$

As suggested by Rivera, ${ }^{16}$ this case may represent a further case allowing the assignment of coloboma to the 22q11-12 interface. Other features in common with the case report by Abeliovich $e t a l^{8}$ include prominent forehead and single transverse palmar crease. The case reported by Abeliovich et $a l^{8}$ also has a number of features in common with other reported cases of distal trisomy $22 \mathrm{q}$ (cleft lip and palate, micrognathia, etc) which was not observed in the present case and may therefore be accounted for by duplication of the region 22q13.1-qter.

The authors wish to thank Mary Strachan and Brian Byrne for technical assistance in this case.

1 Petersen MB, Hansen M, Diernes BW. Full trisomy 22 in a newborn infant. Ann Genet (Paris) 1987;30:101-4.

2 Feret MA, Galan F, Aguilar MS, Serrano JL, Cidras M, Garcia R. Full trisomy 22 in a malformed newborn female. Ann Genet (Paris) 1991;34:44-6. Avirachan S. Anatomic and chromosomal anomalies in 639 spontaneous abortuses. Hum Genet 1980;55:87-98.

4 Eiben B, Borgmann S, Schubbe I, Hansmann I. A cytogenetic study directly from chorionic villi of 140 spontaneous abortions. Hum Genet 1987;77:137-41. 
5 Schinzel A. Incomplete trisomy 22. Hum Genet 1981;56: 263-8.

6 Stratton RF, Dupont BR, Mattern VL, Young RS, McCart JW, Moore CM. Trisomy 22 confirmed by fluorescent in situ hybridisation. Am F Med Genet 1993;46:109-12.

7 Voiculescu I, Back E, Duncan AMV, Schwaibold H Schempp W. Trisomy 22 in a newborn with multiple malformations. Hum Genet 1987;76:298-301.

8 Abeliovich D, Maor E, Bashan N, Carmi R. Duplication of distal 22q. Am ₹ Med Genet 1989;32:346-9.

9 Katafuchi Y, Hashino K, Maeno Y, et al. Partial trisomy 22 with Dandy-Walker malformation. Acta Paediatr fap (overseas edition) 1990:32:566-70.

10 Johnson MP, Greb A, Goyert G, et al. Midtrimester diagnosis and anomalies in the dup(22q) syndrome. $A m \mathcal{F} \mathrm{Med}$ and anomalies in the

11 Bloom SE, Goodpasture C. An improved technique for selective silver staining of nucleolar organizer regions in human chromosomes, Hum Genet 1976;34:199.

12 Fujimato A, Wilson MG, Townder JW. Duplication of the segment q12.2-qter of chromosome 22 due to pater inversion 22 (p13q12.2). Hum Genet 1983;63:82-4.

13 Rivera H, Garcia-Esquivel L, Romo MG, Perez-Garcia G, Martinez Y, Martinez R. The 22q distal trisomy syndrome in a recombinant child. Ann Genet (Paris) 1988;31:47-9. 14 Taylor KM, Francke U, Brown MG, George DL, Kaufhold $M$. Inverted tandem ("mirror") duplications in human chromosomes; inv dup 8p, 4q, 22q. Am 7 Med Genet 1977;1:3-19.

15 Schinzel A, Schmid W, Fraccaro M, et al. The "cat eye syndrome": dicentric small marker chromosome probably derived from a no 22 (tetrasomy $22 \mathrm{pter} \rightarrow \mathrm{q} 11$ ) associated with a characteristic phenotype. Hum Genet 1981;57:14858.

16 Rivera H. 22q distal duplication syndrome. Am 7 Med Genet 1989;34:616. 